\title{
Making Big Data Small: Strategies to Expand Urban and Geographical Research Using Social Media
}

Ate Poorthuis, Singapore University of Technology and Design

Matthew Zook, University of Kentucky

Published as: Poorthuis, A \& M. Zook. (2017). Making big data small: strategies to expand urban and geographical research using social media. Journal of Urban Technology 24 (4), pp. 115-135. https://doi.org/10.1080/10630732.2017.1335153.

Abstract: While exciting, big data (particularly geotagged social media data) has proven difficult for many urbanists and social science researchers to use. As a partial solution, we propose a strategy that enables the fast extracting of only relevant data from large sets of geosocial data. While contrary to many big data approaches - in which analysis is done on the entire dataset - much productive social science work, can use smaller datasets - around the same size as census or survey data - within standard methodological frameworks. The approach we outline in this paper - including the example of a fully operating system offers a solution for urban researchers interested in these types of data but reluctant to personally build data science skills. 
We are facing a "data revolution" (Kitchin, 2015) that is changing the way that researchers study the world, society and cities. While data was once a relatively rare commodity - something to be laboriously collected - technological changes in sensors, mobile devices and data storage combined with evolving social practices have resulted in torrents of data that is big in terms of volume, variety and velocity (Laney, 2001). This represents both a new opportunity (see Poorthuis et al, 2016) and a real challenge for social science research, as we work to understand how big data can help our understanding of the cities around us. In this paper, we present a strategy and platform that enables social scientists interested in working with big data to extract relevant, relatively small data from much larger data sets. As we show, this approach is suitable for many research questions in social science and types of big data as it allows urban researchers to do actual social science without the need to first become full-fledged data or computer scientists.

\section{The challenge of big social media data}

The category of big data is expansive - including sensor data, transactional records, captured images and videos, text, etc. - and has become a critical term within both natural and social science. Of particular interest to the social sciences are big data that come from social media as they represent the day-to-day actions and utterances of ordinary people. This incidental quality of social media is one of the fundamental aspects of big data in that it is almost always secondary data; social media big data already exists out in the world and is not primarily produced or collected for social science research. This means that, instead of having full control over the collection of research data, or being confident that the data meets basic statistical standards, big data often is a dataset of happenstance, rich with potential but messily intertwined with dross. Although data and computer scientists are well versed in the techniques and methods of working with big data, an exclusive focus on data science can problematically devalue the hard-won theoretical and methodological skills of social scientists. This most clearly evidenced by Chris Anderson's (2008) heady declaration of the "end of theory".

Although Anderson's sentiment is often cited as an example of overreach, there are plenty of examples of mischaracterizing of big data, particularly regarding its ubiquity and availability. For example, Arribas-Bel (2014), states:

“...all of these sources are available to researchers without the need to pay any fee or reach exclusive deals with the company/institution providing them. [...] new datasets relating to virtually any quantifiable aspect of human life are appearing" (p.45)

This characterization of easy availability, however, is problematic as the evergreater variety of datasets is certainly not easily or freely available. On the contrary, it is the traditional data sources (e.g. census, local government data) that are most often freely and widely accessible and moreover can also be analyzed with standard or off-the-shelf software and methods. Gaining access to big data often involves paying fees or reaching a licensing agreement and sometimes is not even possible for 'outsiders'. Furthermore, companies who own social media platforms have that goals are not necessarily aligned with academic research. Even if social media data can indeed be accessed, actually collecting and storing such data requires significant resources and skills that can go beyond 
what is traditionally taught within Geography, City Planning or other social sciences. We contend this challenge of access represents an emerging divide between those who are Big Data 'rich' and those who are Big Data 'poor' (boyd \& Crawford, 2012) and thus enabling better access for a wide array of urban related research projects is a key goal. While we focus on the specific case of social media, our proposed solution of "making big data small" could be applied to other types of big data as well.

Accessing big data

In contrast to traditional governmental data sources available via mandate or freedom of information requests, access to social media data depends on corporate policies. Some big data companies make data available to the public through an Application Programming Interface (API) - a defined set of rules and protocols that state how specific data can be accessed or changed - but this not always the case (e.g. Facebook does not provide an API for public or research use). APIs are often constructed because they enhance a platform's visibility, potentially increasing its usage by consumers and developers. Researchers can use the same API to collect data from these platforms but this often require the development of custom software, a skill that most social scientists do not possess. Furthermore, larger data sources (e.g. Twitter) are often only available as a stream. That means data is ephemeral: it is best 'caught' at the moment in which it is produced as it becomes increasingly hard or expensive to access as time passes. For academic use, this 'store it or lose it' principle is tricky; researchers may not know in advance what data exactly to store. Research topics and questions emerge more slowly than the stream of social media, and if data is not captured, access to it becomes costprohibitive or simply impossible. Even if a researcher succeeds in accessing and storing relevant research data, the sheer volume of it can quickly outstrip standard storage capacities and analytic methods.

Within the current literature, there are two distinct approaches to access that can be roughly summarized as more elaborate collection or 'mining' frameworks versus 'ad hoc' data collection. GIScience has engaged with elaborate data collection systems well before the onset of big data as the systematic collection, storage and analysis of large amounts of data has been of long standing concern (Devogele, Parent, \& Spaccapietra, 1998; Han et al., 1997). This earlier work has subsequently been extended to geosocial and big data in systems or 'cyber-infrastructure' focused on the storage and analysis of spatial data in a high-performance and distributed computing setting (Wang, 2010). As a result the number of papers and conference proceedings by GIScientists that study Twitter and its many (technical) facets dwarfs the amount of research that actually uses said data to study 'conventional' urban and geographic phenomena. In other words, the majority of work with big data actually focuses on methods/techniques and is fairly technical in nature (e.g. Croitoru, Crooks, Radzikowski, \& Stefanidis, 2013; Kumar, Morstatter, \& Liu, 2014; L. Smith, Liang, James, \& Lin, 2015), particularly when compared to more traditional research using big data to study cities and society (Baginski, Sui, \& Malecki, 2014; Korson, 2014; Shelton, Zook, \& Graham, 2012).

Although minor implementation details differ, these systems - particularly ones focused on geosocial media collection - are largely designed along the same lines. For example, TweetTracker (Kumar et al., 2014) and GeoSocial Gauge (Croitoru et al., 2013) 
both use the NoSQL database MongoDB, while SensePlace2 uses a Lucene full-text engine (MacEachren, Robinson, \& Jaiswal, 2011). All are designed to collect a stream of social media data (Twitter in these cases) that matches a pre-defined set of keys as opposed to the entire stream of data. It is up to the researcher to decide - up front - what is and what is not relevant to collect. TweetTracker and Senseplace 2 were specifically designed as tools for use in disaster response and receive a moderate amount of attention from researchers beyond the initial authors (as judged through citations and adoption in published work). Many other systems do not fare as well and seem to be primarily a proof of concept or prototype that illustrates that such data can indeed be captured and analyzed. Many of these prototypes outlined in the literature also suffer from issues where the actual implementation is very short on details (Kumar, Barbier, Abbasi, \& Liu, 2011), not very feasible in practical research cases (Huang \& Xu, 2014) or not very extensible to application beyond the limited scope of a test case (Fujita, 2013). This inability to extend elaborate data collection systems has limited their effectiveness for the research community more generally. Even the Library of Congress, tasked with archiving every tweet ever produced, has not yet succeeded in making the Twitter archive easily accessible and usable for academics - despite consulting from the private sector (Leetaru, Wang, Cao, Padmanabhan, \& Shook, 2013).

As a result, many researchers instead turn to more 'ad hoc' data collection via the open source API libraries and plugins to social media that exist for virtually every programming language. Although some researchers use (commercial) third-party services (Kounadi, Lampoltshammer, Groff, Sitko, \& Leitner, 2015) or an existing collection system (Crampton et al., 2013; Stefanidis et al., 2013), most simply leverage available and relatively simple open source tools. For example, within Geography, TwitterHitter (J. White \& Roth, 2010) was developed by cartographers and allows the researcher to both collect and visualize data in a cartographically-sound manner. Outside of Geography, the Excel plugin NodeXL (M. A. Smith et al., 2009) is popular. The plugin allows any researcher to collect social media data and store it in a simple database, flat text files or spreadsheet. This is a feasible strategy because in most cases the resulting datasets are rather small. For example, over 80 percent of Twitter studies use datasets that are smaller than 10 million records (Weller, 2014; Zimmer \& Proferes, 2014), which is not at all 'bigger' than more conventional data sets in quantitative social science. These datasets easily fit within the memory of even a modest laptop without the need for special big data tools or software. Some datasets are even smaller, for example Yang's (2015) study of depression in Twitter users uses a randomly sampled 402 tweets - only around 50,000 characters of total data. Although size-wise this seems very small compared to the vast pool of billions of records available, just because data exists does not mean it needs to be included in an analysis. Korson's (2014) qualitative analysis of 407 tweets around UN peacekeeping missions shows clearly that there is value in Twitter data regardless of the total number of records.

While the ad hoc approach makes big geosocial media data more accessible for research it suffers from two key weaknesses. First, the ability to study a phenomenon longitudinally requires a researcher to maintain a constant always-on data collection effort. Many such efforts are deployed within a desktop environment, which is difficult to maintain over the long term. Weekends, vacation periods and power cuts all confound this kind of setup and depending on the collection parameters, gathering data can fairly quickly 
fill a hard drive and overwhelm existing backup systems. The second weakness is the lack of standardization or documentation about data collection methods. While some of this work is very explicit about its collection strategy and implementation (L. Li, Goodchild, \& $\mathrm{Xu}, 2013$; J. Lin \& Cromley, 2015), others are rather opaque (Kay, Zhao, \& Sui, 2014; Widener \& Li, 2014; C. Xu, Wong, \& Yang, 2013). This uncertainty is problematic as the specific collection strategy can have a large impact on the resulting data and research outcomes and thus any opaqueness increases uncertainty about outcomes. As just one example, Morstatter et al. (2013) conducted an in-depth comparison of data acquired from the freely accessible 1 percent sample of Twitter data versus the very expensive 'firehose' that includes 100 percent of all tweets and find several sources of bias within the sampled data ${ }^{1}$.

\section{Using big data by making it small}

In short, efforts to use big data in urban research questions can quickly get bogged down in mechanical hurdles that are beyond the traditional skill set of researchers. This need not be the case and we argue for the need to develop approaches for working with big data that does not require every individual researcher to develop skills more akin to computer science than social science. While there is no single best approach, we outline a technological solution - including a fully operational system - that provides social scientists with access to big social media datasets without the need to become programming experts. This solution, based at the University of Kentucky and called Data on Local Life and You (or DOLLY), leverages the robustness and scalability of a serverbased data mining system on the back-end, with the usability advantages of ad hoc desktop applications and the convenience of small, relevant datasets that minimizes technical barriers.

As an ongoing test case (and research tool) DOLLY has collected all geotagged tweets in the world, going back to June 2012, and enables real-time search and extraction from its corpus of $12+$ billion tweets. Twitter is a useful test case as it is one of the most prolific social media platforms and while this paper focuses exclusively on the specifics associated with Twitter data collection, DOLLY is designed to ingest smaller geosocial media datasets without a problem. The exact same architecture and resources can also be used to add additional social media platforms (e.g. Flickr, Instagram, Foursquare) to the system relatively easily.

Paradoxically, a key attribute of DOLLY is its ability to make big data small and allowing researchers to quickly extract only the relevant data. While many existing big data approaches perform analysis on complete datasets spanning terabytes, in practice, most social science work based on Twitter data source uses relatively small datasets similar in size to other more conventional data sources such as census data. While such datasets might be relatively large, especially when compared to studies that only look a few census tracks or counties, these research projects simply have little use for big data methodologies

\footnotetext{
1 Particularly relevant for urbanists and geographers is that, for the subset of geotagged tweets, the difference
} between the sample and the firehose is negligible. 
used within data science to review datasets in their entirety. DOLLY is designed to allow researchers to narrow down the universe of tweets quickly to a relevant dataset that measures in thousands or tens of thousands, not millions or billions. In the same way that word-processors allow users to focus on writing text, DOLLY abstracts away the computer science and allows a social scientist to do social science.

\section{Outlining the DOLLY approach}

Given these current collection methods, our approach with DOLLY collects and stores all geotagged tweets as they are produced and are delivered in Twitter's data stream and does so in a way that is consistent, reliable and minimizes collection bias by making sure that all data in the world is collected. This is the big part of the data collection. However, and most importantly, after initial collection and storage, DOLLY is designed to make it easy for any researcher to search through billions of data points and extract only the data that is germane to the research question. This approach effectively gives urban researchers quick access to the small and relevant datasets that are needed for the vast majority of research question, without any of the barriers to access or reliability issues surrounding ad-hoc data collection. While our intention is to share the code developed for DOLLY the challenges of maintaining the system - particularly the means to extract small datasets - have delayed finalizing a clean and properly annotated version that would be useful and coherent to others. In the meantime we make the data and system available for qualified researchers on a per-request basis. In the following section, we will provide both details on the exact approach used and discuss several lesson-learned during the development of the project.

\section{Complications with terms of service}

An ongoing issue associated with Twitter data (or any data sourced from a private company) are the Terms of Service (ToS) - to which every API user has to agree - and that are continuously updated and changed. For example, earlier versions of the ToS prohibited sharing data collected through the streaming API but under the current ToS (Twitter, 2015 ) it is possible to share collected data up to 50,000 records per user per day as long as that process is not automated. The Twitter ToS also requires looking for deletion events in the stream so that if a user deletes a certain tweet, it would also be deleted from stored records as well. In short, while ToS requirements are framed as legal issues there are a number of methodological challenges and ethical questions as well. For example, simply checking for deletion events and acting on this information, introduces a whole new level of operational complexity.

Although Twitter has cracked down on public websites and commercial services that share data ("Export Twitter Followers and Friends using a Google Spreadsheet," 2011; "Removal of Export and Download / API Capabilities," 2011; "The story of getting Twitter data and its 'missing middle' | ScraperWiki," 2014), many academics have shared Twitter data for replication and further research (e.g. Dooms \& De Pessemier, 2013) and Twitter has so far been quiet on the matter. This makes the Terms of Service and how best to follow them somewhat of a gray area for academics, particularly as the ethos of information technology companies has been steeped in the academic traditions of knowledge sharing and research using Twitter data serves as free PR for the company. Thus, it seems that in 
some cases - particularly for non-commercial applications -the "hacker ethics" mentality (Levy, 1984) seems relevant, although there is always potential for it to be trumped by a more hardline and legalistic stance by Twitter.

\section{Leveraging Twitter REST and streaming APIs}

Until 2009 Twitter only provided a so-called REST API. This API allows developers to build third-party applications that interface with Twitter using HTTP verbs (GET, POST, DELETE etc.) to specific 'endpoints'. For example, a GET request to / $\mathrm{fr}$ i ends / ids would get all the friends of a specific user, while a POST request to / s tatuses / upda te would send a new tweet. This allows a developer to build an application on top of Twitter, or even to build a Twitter client that mimics or improves on Twitter's own web interface and smartphone apps. The structure of REST API can be used to search Twitter's database for tweets that match certain criteria, although Twitter has constrained the search to the last seven days in history and a maximum result set of approximately 3500 tweets. Excessive use and polling of this endpoint plagued Twitter's overburdened servers during its early years so the company instituted a 'rate limit' on the number of times a person could query an endpoint within a certain timeframe - making it less feasible to use in the collection of larger datasets.

Twitter was originally very welcoming to researchers and allowed them to be white listed to get a higher rate limit for academic research. It discontinued this program once the monetary value of its data became clearer. In 2009, Twitter released a Streaming API to make collecting some data a bit easier. Unlike the REST API, which actively needs to be polled at specific intervals to get new data, with the Streaming API opens a long-lived connection (a stream) to Twitter's servers that receives updates whenever they are being created. This is analogous to a mail client checking mail at specific intervals (a REST API request) versus the push notifications on modern platforms that are sent as the new mail arrives (a Streaming API always-on connection). There are three different streaming levels available. The standard streaming level contains roughly a 1 percent sample of all tweets and can be filtered by specific keywords, user ids or geographic bounding boxes. If filtering is enabled, only those tweets matching the filter will be returned. Importantly, if the tweets matching the filter amount to less than 1 percent of all tweets, all tweets matching the filter will be returned instead of a random sample. An elevated access called the gardenhose contains 10 percent of all tweets and was offered to academics free of charge in the early years of Twitter's operations ${ }^{2}$. DOLLY was grandfathered into gardenhose access level in 2011 but new applications from academics are now referred to Gnip, a private company where such access can only be bought. Finally, there is a firehose level of access that contains all publicly available tweets but this level is seldom given out and is cost-inhibitive for most research projects (Morstatter et al., 2013).

\footnotetext{
2 The DOLLY project received an academic white listing in May 2009 for a different project (Dugundji, Poorthuis, \& van Meeteren, 2011; van Meeteren, Poorthuis, \& Dugundji, 2009). This original white listing allowed DOLLY to access the elevated garden hose (10\%) streaming access without going to a third-party commercial vendor in 2011 .
} 
The principle downside of streaming for research purposes is the nature of streaming itself. The 'use it or lose it' approach means that a stream needs to be set up before an event of interest occurs and when, for example, a researcher later realizes a certain keyword is pertinent to the research at hand it cannot be retroactively added to the dataset. As such, with the Streaming API it is especially important to cast a wide net and collect as much data as might be possibly needed in the future. In addition, many topics of interests are tweeted about infrequently and it can take many months or years before a 'large enough' dataset is collected for specific topics or areas under study.

The goal of the DOLLY system is to collect every single geotagged tweet and to do so, it uses both the 1 percent as well as the 10 percent Streaming API. It sets up separate, parallel streams at the 1-percent level that are each filtered by a different spatial bounding box covering a continent. Each of these streams can collect 1 percent of tweets - if the filter on a stream matches more than 1 percent, the result set will be sampled down to 1 percent. Thus, by narrowing each stream to a relatively small bounding box, we ensure that the geotagged tweets that fall within this box are (almost) always below 1 percent of all tweets.

The gardenhose access level collects a separate stream consisting of 10 percent of all tweets, not just the subset of geotagged tweets. This is done to monitor the total amount of tweets sent everyday, as well as determine what percentage of those tweets are geotagged. As is shown in Figure 1, the total amount of tweets sent each day varies between 200 and 400 million messages per day. Although Twitter, around their IPO in 2013, enthusiastically reported that 500 million tweets a day were sent (Twitter, 2013), they have grown increasingly silent on the exact number and it seems likely that the number of tweets per day has indeed stabilized around or below 400 million (Leetaru et al., 2013; Oreskovic, 2015). Despite the increasing number of GPS-enabled smartphones, the total share of geotagged tweets is relatively stable as well, around $2.5 \%$ of the total (see Figure 2). As such, DOLLY captures around 10 million geotagged tweets worldwide everyday via the seven parallel continental bounding boxes using 1 percent streams. Although the number of tweets per day itself is relatively stable, their geographic origin is constantly evolving. For example, since DOLLY began collecting data in 2012 some countries in the Global South have emerged as more prominent on Twitter so that by 2015 Twitter has clearly evolved beyond exclusive presence in rich, English-speaking countries. The linked ranking in Figure 3 shows the relative share of tweets for each country in 2012 and 2015 to show how the contours of Twitter use has evolved over time. 


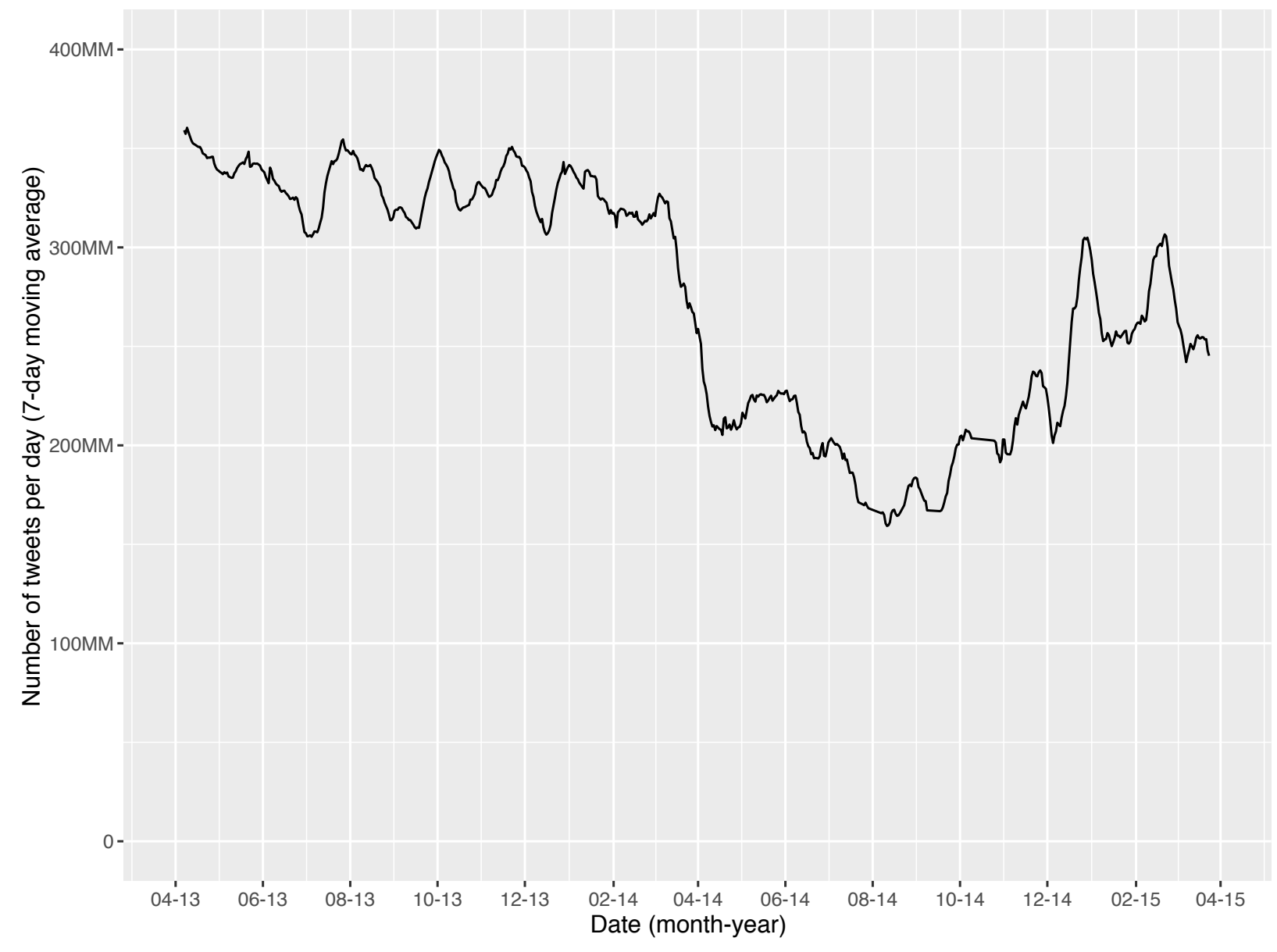

Figure 1, Tweets per day in the $10 \%$ sample 


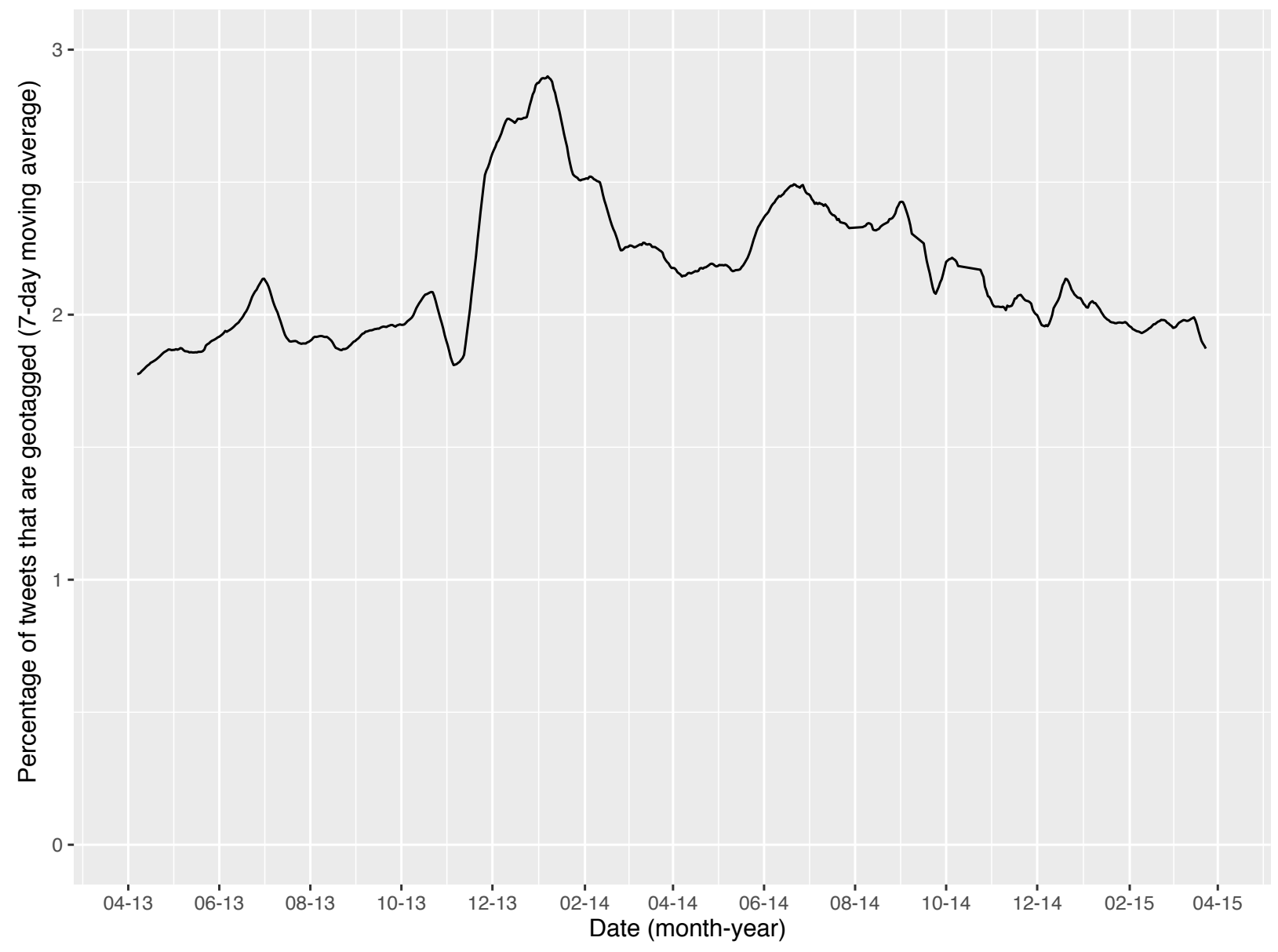

Figure 2, the relative share of geotagged tweets ${ }^{3}$

3 The fluctuations seen in Figure 1 and Figure 2 are not a result in the DOLLY methodology or system and are tied to changes in (1) actual Twitter usage and/or (2) changes in Twitter's public API. However we have been unable to clarify with Twitter the exact cause of these changes. 


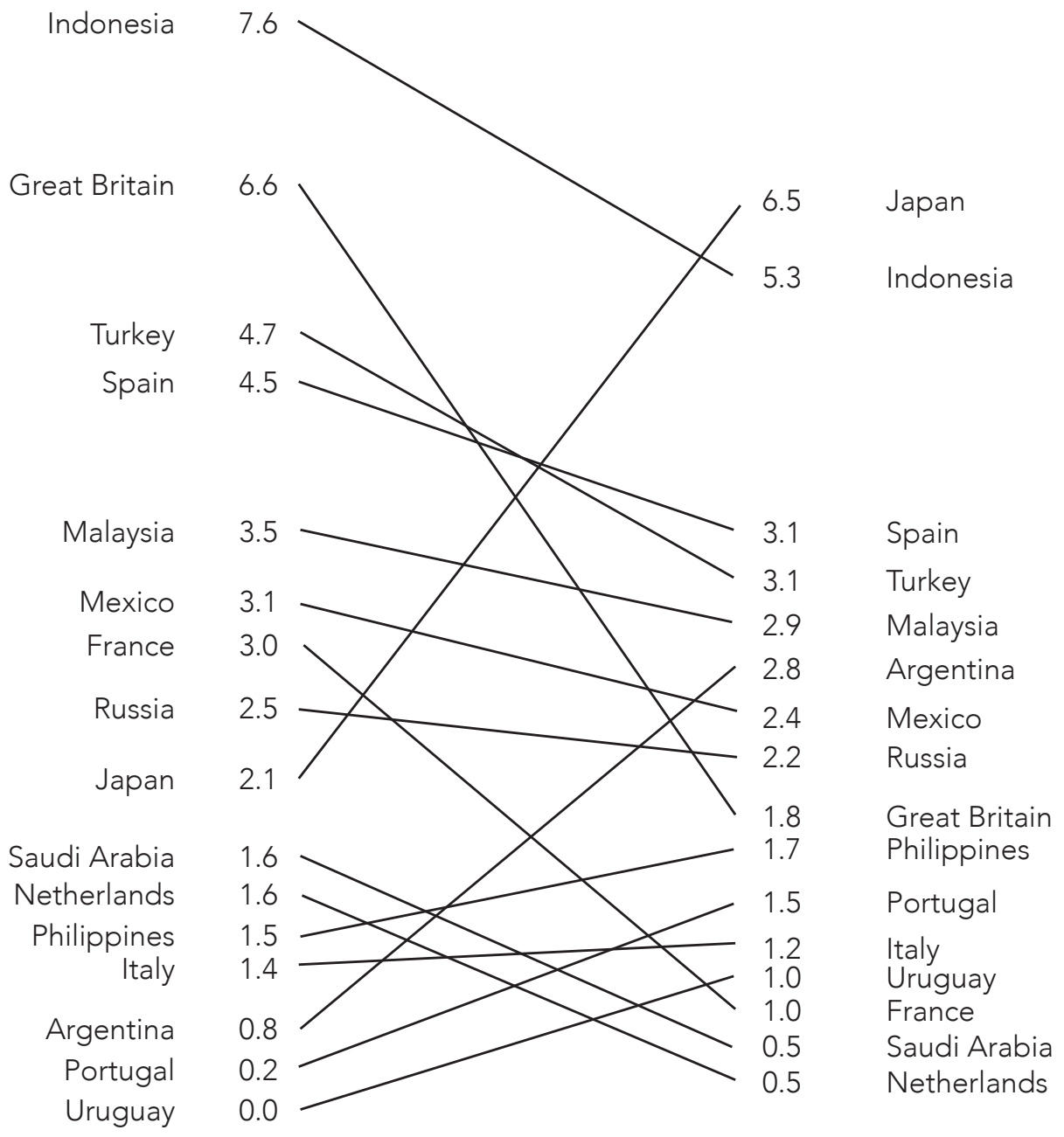

Percentage of tweets sent from each country (relative to all tweets worldwide) for most active countries on Twitter. Change from 2012 to 2015. 


\section{Figure 3, Change in relative share per country between 2012-2015}

To set up these streams, worker scripts ('streaming workers') - written in Ruby using the well-supported open source Tweetstream library (tweetstream, 2015) - connect to Twitter and listen for incoming tweets. Each worker runs on a separate virtual machine in a virtual private cloud at the University of Kentucky. This minimizes the impact of a potential outage in any one machine as virtual machines can be moved to different physical machines when an outage occurs. Furthermore, each of the streams is monitored by a separate service that continuously checks if the stream is still operating correctly and, if not, attempts to restart the process ("Easy, proactive monitoring of processes, programs, files, directories, filesystems and hosts | Monit," n.d.) If a worker script or other process is repeatedly failing or are not running for several minutes, email warnings are sent to enable quick human intervention. Apart from a few inevitable power outages, network configurations errors and related issues ${ }^{4}$, this has enabled a continuous and uninterrupted consistent collection of all geotagged data since June 2012 .

\section{Selecting a data format}

Tweets arrive from the Streaming API in the JavaScript Object Notation (JSON) and are kept in that format throughout the collection processes. Upon arrival a tweet is submitted to a queue ${ }^{5}$ for further processing. Queuing systems are used in many applications, including finance and high frequency trading to track and process transactions in which speed and performance of message sending and delivery is of the essence. Here, we use a messaging queue specifically to decouple the initial collection and the subsequent filing away of tweets. As Twitter activity is spiky, for example during a Superbowl event, tweets can sometimes stream in faster than they can be filed away. The queue functions as a buffer and can smooth out peaks without the risk of losing tweets because the storage process cannot keep up. The queue runs on two mirrored virtual machines to enable high-availability failover.

With smaller amounts of data it would be sensible to store data in text files or a simple relational database, but this does not scale well to a continuous multi-year collection of data of this magnitude. Each day, 10 million tweets of raw data means about 8 GB of data (after compression) needs to be stored. This translates to about 9TB of data storage for the 2012 to 2016 dataset, not taking into account backup and redundancy. As such, storage of such datasets is not a trivial matter and needs to be carefully designed to

\footnotetext{
${ }^{4}$ Although the University's data center was outfitted with its own power generator and UPS the system was impacted by power outages. Likewise, some configuration errors and software bugs resulted in some gaps (generally of a few minutes or hours) while updates were preformed. While we discussed filling these gaps the combination of a short time horizon for action (before Tweets were no longer available), the weight of other demands on our time and lack of human resources made filling these gaps a lower priority than other tasks crucial to keep the system going. While DOLLY was up approximately 99.99 percent of the time these gaps bring home the difficulty in maintaining $100 \%$ uptime in long-term data collection.
}

${ }_{5}^{5}$ The open source RabbitMQ that utilized the open AMQP standard is used here (Vinoski, 2006) 
match both current and future needs. In the initial implementation phase of DOLLY, Amazon's cloud service was used to host database servers as well as storage and backup of data. However, in 2011 Amazon's virtual machines had notoriously slow IO operations compared to dedicated servers and the database had trouble writing the amount of data to disk fast enough (Hill \& Humphrey, 2009; Juve, Deelman, Berriman, Berman, \& Maechling, 2012; Juve et al., 2009; Ostermann et al., 2010). Furthermore, running multiple virtual machines as well as storing large amounts of data can get expensive quickly; especially since Amazon's cloud service is based on recurring monthly costs. Therefore, after initial testing we invested in servers and storage space to build our own private cloud as it was much more economical than a recurring payment to a cloud service provider. It also ensures the continued availability of the system and data within after the collection effort or the entire project ends as the majority of costs are not incurred on a monthly recurring basis but are paid upfront when hardware is bought.

Once a hardware configuration is selected, the next key question is the file format used for storage. During the initial implementation of DOLLY in the fall of 2011, all tweets were stored as compressed JSON text files. Once JSON is compressed, it is remarkably space-efficient, convenient and robust: data corruption is less of an issue with text files; they can be read by any software program; and tweets can be bundled per day and archived away in off-site storage if and when local storage space becomes an issue. This is exactly the way the Library of Congress is storing their Twitter archive (Congress, 2013) and is also how Mapbox is storing a similar archive (Fisher, 2014). However, when one wants to analyze data, the compressed JSON format becomes a major inconvenience. Even to extract a small, relevant dataset of 1,000 tweets that match, say, a certain keyword, the entirety of the data needs to be read and combed through. On a single server, this can take many days - which is exactly why many big data companies use distributed systems like Hadoop (T. White, 2012) or other map-reduce frameworks (Gates et al., 2009) that fan out chunks of the data to be individually processed by a large set of machines (the map stage) and, when done, the results are combined back on to one single machine (the reduce stage).

\section{Relational, NoSQL and Elastisearch databases}

Thus, while implementing a large cluster can speed up access, map-reduce frameworks require a significant amount of skill and runs counter to the goal of increasing access for non-technical social scientists. Other relational database solutions such as MySQL and Postgresql can also be used to store large amounts of data, but they are not optimal for big data research two reasons. First and foremost, they require the researcher to design in advance a specific schema - the data fields and their types (integers, text etc.) for the dataset. This makes adding and querying data much more efficient but, as is the case with social media like Twitter, the incoming data stream can often change its format, adding or removing fields and changing data types without notice. Thus incoming data could not be added unless the researcher engages in an ongoing cat-and-mouse game of restating the schema and converting old data to the new format. Second, relational databases, being a much older technology, are often built with single-server architecture in mind. Although they can potentially distributed across servers (an absolute necessity given the scale and growth of data), these configurations are often fragile and require significant system administration. 
For these reasons, DOLLY initially used CouchDB (J. C. Anderson, Lehnardt, \& Slater, 2010) for the underlying data storage and then switched to MongoDB (Chodorow, 2013) the latter being a widely used solution for other big data collection systems (Croitoru et al., 2013; Kumar et al., 2014). These are NoSQL databases and contrast to the relational database approach, where each data observation is structured as a row with a set number of fields, and instead use a document model. In NoSQL databases, each observation is a 'document' (rather than a row) and the fields in each document can be completely different from one another. Furthermore, fields themselves can be nested, containing additional fields as children. This structure fits neatly with the way tweets are natively stored (JSON) and some NoSQL databases even use JSON in their underlying model.

These NoSQL databases are designed with big data in mind, i.e., scalability is a key concern, and should theoretically work with many terabytes of data in a distributed cluster (Abramova \& Bernardino, 2013; Cattell, 2011; Pokorny, 2013). In practice, however, MongoDB suffered from reliability issues, data corruption and scalability that required constant and vigilant system administration. Furthermore, as the historical data kept growing, MongoDB had escalating difficulty keeping up with the ingestion rate of Twitter data collection and combined with cumbersome cluster maintenance procedures meant a significant investment in system-administrator-type skills.

Given these short-comings, we converted the DOLLY system in the Spring of 2012 to use ElasticSearch (Gormley \& Tong, 2015) as its primary database store and querying layer. ElasticSearch is a noSQL-like scalable, distributed database that supports full-text search (built on top of Lucene), has very solid geo-spatial support, and can handle changes in the structure of inserted data on-the-fly. Furthermore, it compresses data efficiently (requiring less storage space) and scaling up by adding additional nodes is turnkey: when a new node is added, all data is automatically distributed evenly over the cluster.

Furthermore, it has built-in redundancy by duplicating data one or many times across the database, increasing the speed with which data can be retrieved but also making the entire cluster more resilient.

DOLLY initially ran two ElasticSearch virtual machines with 500GB storage space and 4GB of RAM each with additional nodes easily added when needed (or funding became available). Over time, the storage layer for DOLLY grew to a total of 33 nodes with 1.1TB of storage and 10GB of RAM each. Data for the most recent 12 months, which is queried and analyzed more often, is replicated twice (thus stored three times in total) and older data is replicated once. To ensure longevity of the dataset, a back-up of the entire dataset is run on a daily basis to a storage facility in the same building as the private cluster and once a week, a back-up is made to an offsite facility.

\section{Adding metadata variables}

Before data is inserted in the ElasticSearch datastore, a number of operations are performed by a set of worker scripts (each running on a separate node) that read from the messaging queue where tweets were deposited by the streaming scripts. Most importantly, the geotag of each tweet is evaluated. There are three primary types of geotags attached to tweets: one that is a relatively accurate latitude/longitude coordinate pair generated by a smartphone (either through GPS or cell tower and Wi-Fi location); one that is a 'place', 
something like 'Brooklyn, NY' or 'USA'; and one that has both a place and a coordinate pair (see Poorthuis, et al, 2016). Furthermore, the latitude/longitude coordinate is used to perform a spatial join to larger areal units: country and state/province-level for all countries $^{6}$ and the addition of county and census tract levels for the US. This makes searching for data for an entire country or a specific census tract (within the US) much faster later on.

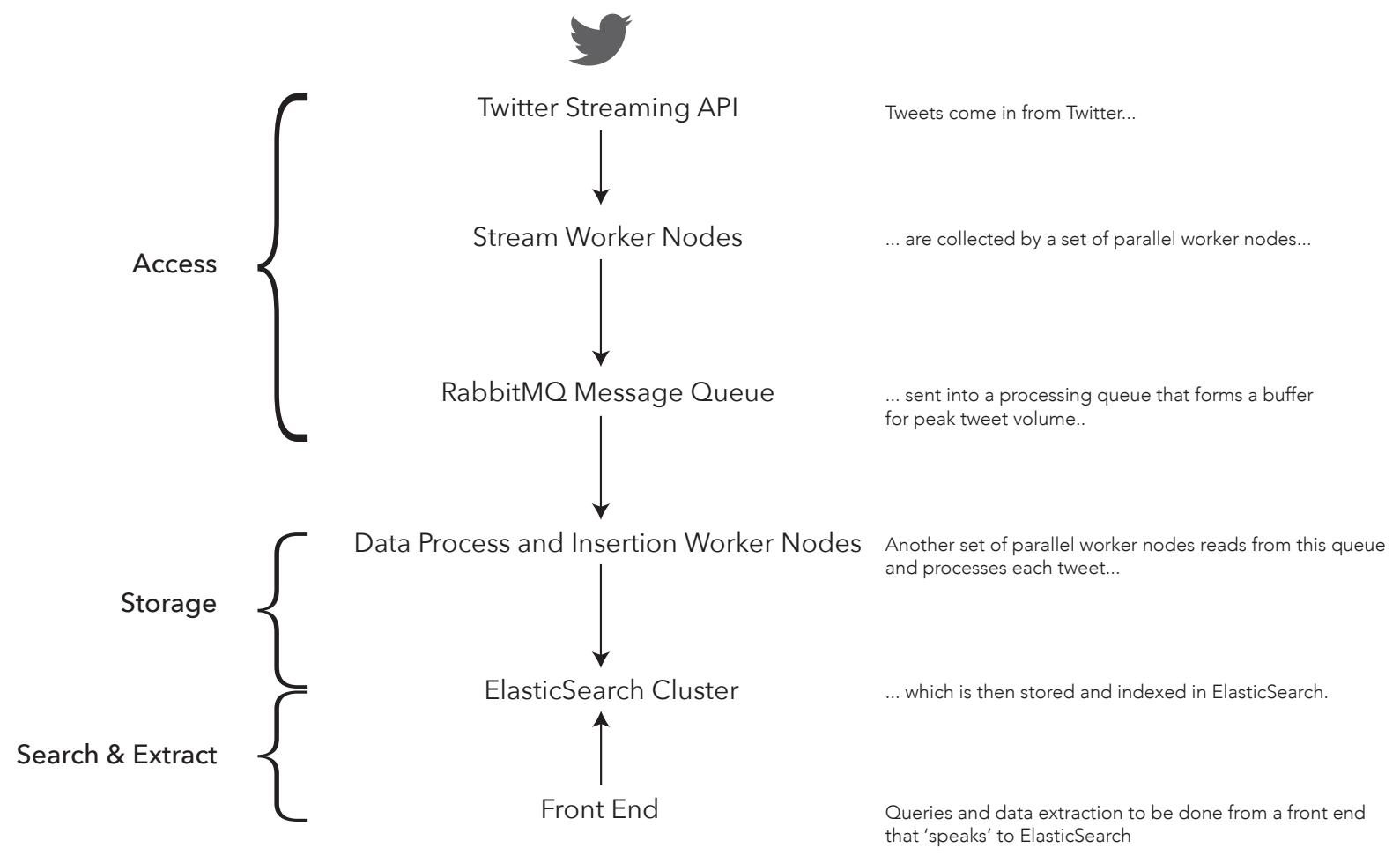

Figure 4, Schematic overview of DOLLY system

The final piece of metadata added is a random number between 0 and 100 million to allow a researcher to take fast random samples post-hoc. This is particularly helpful for many research questions (particularly during exploratory stages), as often one only needs to analyze a random sample. Databases, however are often not designed to allow for fast random sampling at query time and thus doing so post-hoc can be expensive (Olken \& Rotem, Pokorny 1990) and the addition of a random number allows for a cheap range query on that field: a query matching all numbers smaller than 10 million will then result in a random $10 \%$ sample of all data. Both the geographic and random number metadata are added to the tweet document before inserting into the ElasticSearch database (see Figure 4).

${ }^{6}$ Using Natural Earth Data ("Natural Earth," n.d.) and the PostGIS spatial database (Ramsey, 2005) 


\section{Indexing for search and extraction}

Once data is collected and metadata added, the most important feature for social scientists is to ensure that search and extraction functions can be performed quickly. Many databases rely upon an indexed key for searching with the goal of adding index data as quickly as possible as well as keeping creating indices to a bare minimum as index construction is time and CPU expensive as well. This means extraction of data in nonanticipated ways -- searches on non-indexed fields -- becomes very 'expensive' as the entire data set needs to be looked through.

The design of DOLLY takes the opposite approach: as computing power is relatively cheap, as much data is indexed as possible and this is done immediately when tweets come in. This means that when a search is actually conducted it can be searched very quickly across virtually all fields. In practical terms, this means that the entire cluster (33 ElasticSearch nodes, 8 worker nodes) is continuously indexing data as it arrives, for fast extraction via search later. Thus, a search for some specific data from 2013, leverages computing cycles completed years earlier, invisibly shifting the time-space of computer processing to the advantage of the researcher.

Instead of tables, ElasticSearch uses indices that in the case of DOLLY are organized by month and the seven bounding boxes (i.e., continents) used for the Twitter Streaming API. This both allows data to be managed on a month-by-month basis (for example, decreasing replication for indices older than 12 months) as well as also further optimizing queries. For example, to search and extract data from a specific continent and month, one only needs to access that month's index, twitter_na_2013-01, and a wider search can be done via wildcards, searching in 'twitter_na_2013*' searches all indices for North America created in 2013.

Within each index, every single field is individually indexed. For fields that contain text (such as the actual tweet text and the user bio) a full-text index is constructed by splitting the field into terms and creating n-grams - strings within each word up to the length $\mathrm{n}$ - for each term. For example, a 1-gram or unigram of the word 'Dolly' would yield ['D', 'o', 'l', 'l', 'y'], a 2-gram or bigram would yield ['Do', 'ol', 'll', 'ly'], and so on. This allows searches to match partial words and to search using wildcards (p?zza*) and even do fuzzy string matching using Levenshtein's string distance (Levenshtein, 1966) to account for spelling variations, singular-plural, shortened words and general typos.

The coordinates of each tweet are spatially indexed as well so that, if needed, spatial queries can be done based on bounding boxes, polygons or even a distance from a certain point. ElasticSearch also calculates a geohash at multiple scale levels ("Geohashes," n.d.). A geohash was originally designed to express a location in URL-friendly format but is also a very effective way of aggregating locations up to multiple scale levels. At the coarsest level, geohashes divide the world into 32 cells (each about 5000 by $5000 \mathrm{~km}$ ), each expressed by one letter or number (e.g. ' $\mathrm{g}$ '). The second level divides each cell in 32 sub-cells, which can be expressed by a two-position hash, e.g. 'gq'. The final level 12, where the hash has a length of 12, e.g. 'gq5h9z2ng91a', has a precision of just a few centimeters. Having each point pre-indexed in this way, also allows DOLLY to quickly aggregate individual tweets up 
to grids of varying scale and compare spatial distributions of different queries at different scales in a fast manner.

\section{Putting DOLLY to Work - User interfaces and access}

The final piece of the system is a series of user interfaces that provide query and extraction access for a range of different skill levels. Researchers comfortable with coding can interface with Dolly's back-end directly through http requests sent from their programming language of choice or from within the statistical language $\mathrm{R}$. We have also developed a light front-end website that allows full access to a boolean search logic, a preview of the tweets in the search results and an export of the resulting data to CSV. Within this interface, users could submit queries ranging from 'give me a random sample of 50,000 tweets sent from New York City in the last 12 months' to more complex ones that use fuzzy matching and wildcards on words in either the tweet itself or the user biography. Thus, even non-coders have a significant and compelling level of search and extract access. While lower level querying - via R or some other programming language - allows for automated queries, data processing and even visualization, this web based solution fulfills our goal of providing non-programmer access to a comprehensive archive of all geotagged tweets sent since June 2012 .

A remaining challenge for future development is expanding the system's front-end to allow for a more user-friendly experience for preliminary data visualization. In its current implementation, analysis of the data beyond a first cursory look at tabular data requires export to a desktop GIS or statistical environment rather than housing the first steps of exploratory analysis completely and reliably within the browser application. This would provide researchers an immediate insight into the underlying spatial pattern at hand, allowing for a quicker iteration in the research cycle and be especially fruitful in the early stages of research projects where ideas, hypotheses and keywords are under evaluation.

At present the main bottleneck facing DOLLY is that we lack the human resources to effectively administer and secure access to it. As a result direct access is limited to those within our research team, but we do work with qualified researchers who contact us directly or via the DOLLY webpage to extract data for specific projects. Thus far, however, we have not broadcast this availability widely primarily due to personnel and time constraints (we have to retrieve the data ourselves) rather than technical or legal limitations. The most recent ToS from Twitter clearly specifies the number of records that can be returned - 50,000 per user per day - which seems a workable dataset size for many social science questions. In short, DOLLY is not just a prototype of a big data collection and analysis system (cf. Fujita, 2013; Huang \& Xu, 2014) but a fully implemented platform.

This includes the use of DOLLY by approximately a dozen researchers at institutions worldwide. Based on our input, these scholars have used datasets to study topics ranging from examining urban displacement (Schaefer, 2014), to tracking the view sheds of wild fires (Klein, 2014), to analyzing political and cultural discourse (Jung, 2015; Chew, 2016), to studying the relationship between fast food and public health (Christian, 2014) as well as a number of doctoral dissertations within human geography (Shelton, 2015; Stephens, 2012). DOLLY data is also currently in use with several ongoing research projects at UC 
Santa Barbara, the University of South Carolina, the University of Amsterdam and the University of Twente in the Netherlands ranging from hate speech and public health to the spatial dispersion of knowledge.

In addition we have used DOLLY to search and extract relevant data for our own articles (Crampton et al., 2013; Poorthuis \& Zook, 2014; 2015; Poorthuis, Zook, Shelton, Graham, \& Stephens, 2015; Shelton, Poorthuis, Graham, \& Zook, 2014; Zook \& Poorthuis, 2014). To provide insight on the data retrieval and analysis process we offer a brief review of one of our projects (Shelton, Poorthuis, \& Zook, 2015) to show how DOLLY can be put to work. This particular analysis engages with wide-spread understandings of mobility and segregation in Louisville, KY and uses big geosocial media data as an indicator of the mobility of different parts and populations of the city. More specifically our research question was whether we could show that individuals from the West End (a predominantly African-American and poorer neighborhood) inhabited and moved in different parts of the city than those from the East End (a more well-off and predominantly white part of town). Beginning with an initial set of 5.7 million geotagged tweets (the entirety sent in Louisville from June 2012 to July 2014) we filtered down to only the 1.5 million tweets sent within the neighborhoods we studied. Using this subset we next identified individual users who (1) had at least 40 tweets in our data and (2) had sent the majority of tweets from one of these two neighborhoods. This yielded 703 East End and 662 West End individuals sending at total of 274,338 and 398,432 geotagged tweets respectively. In order to control for 'power users' who are responsible for a disproportional share of tweets, our final step was to sample down for each user so that our final database for analysis was approximately 100,000 tweets from these 1,365 users, a reduction of approximately 50 times the initial set with which we started.

In this example, the ability of DOLLY to shift from "big" to "small" data allowed us to "go beyond the geotag" (Crampton et al, 2013) and study "the fundamentally fluid, networked and relational nature of places in the city, as well as the dynamism of how people live in and occupy these places" in order to "build upon our understandings of how this data can reveal a more complex and nuanced set of socio-spatial relations than is typically assumed." (Shelton, Poorthuis, \& Zook, 2015: 210). While we remain cautious about the applicability of social media and other types of big data analysis to our research (see also Goodspeed, 2013; Zook, 2017), we also see these data as a means to engage with popular but unsubstantial understandings of society and space (see also Schweitzer, 2014). In short, the DOLLY system - along with other similar approaches to big data - provide the means for social science to successfully engage with the fast growing application of big data to the world.

\section{The future of big geosocial media data research}

This article outlines a solution for making big data more accessible for social science and urban research through a counter-intuitive approach that works to make big data small. While not appropriate for all research questions - for example, data science approaches that seek patterns across the totality of a phenomenon - it is extremely useful for a range of geographic and urban research. Particularly because it allows scholars who do not have the background or computing infrastructure necessary for collecting and maintain social media datasets to access and use these data. Moreover, by collecting all 
social media data (in this case geotagged tweets) a priori, it provides researchers with the flexibility to form research questions and hypotheses within established workflows rather than under the pressure of time, fearing that data about a particular phenomenon will "time out" and become unavailable.

Of course, gaining access to data is but the first step of any research project and care must be exercised in how it is visualized and analyzed. This is especially the case for scholars using social media data for the first time or those unfamiliar with the details of spatial analysis (Zook, 2017). In the case of the former, real care must be taken to ensure that a social media event (a post, a tweet, etc.) is interpreted carefully and broadly rather than simply assuming that it has only one meaning (Crampton et al, 2013). While sentiment analysis provides some metrics on the meaning of a social meaning utterance, the use of slang and the small size of individual social media events confound overly naive interpretations. For those new to spatial data it is important look beyond clustering of events, as these often are primarily stories of population densities. Rather, proper normalization of the data as well as decisions about the type of areal units to be used are essential when using social media data (see Poorthuis et al, 2016 and Zook, Poorthuis and Donohue, 2016).

Despite these cautions, the case study of Louisville (Shelton, Poorthuis, \& Zook, 2015) clearly shows the applicability of using big geosocial media data to understand cities. Thus, DOLLY offers a way forward to use social media (as well as other big data sources) in social science research that can successfully overcome the technical challenges of big data and allow researchers to focus on their theoretical and methodological expertise rather than the techniques of system administration.

\section{References}

Abramova, V., \& Bernardino, J. (2013). NoSQL databases: MongoDB vs cassandra. the International $C^{*}$ Conference (pp. 14-22). New York, New York, USA: ACM. http://doi.org/10.1145/2494444.2494447

Anderson, C. (2008). The End of Theory: The Data Deluge Makes the Scientific Method Obsolete. Retrieved August 14, 2015, from http://archive.wired.com/science/discoveries/magazine/16-07/pb_theory

Anderson, J. C., Lehnardt, J., \& Slater, N. (2010). CouchDB: the definitive guide. O'Reilly Media, Inc.

Arribas-Bel, D. (2014). Accidental, open and everywhere: Emerging data sources for the understanding of cities. Applied Geography, 49, 45-53. http://doi.org/10.1016/j.apgeog.2013.09.012

Baginski, J., Sui, D., \& Malecki, E. J. (2014). Exploring the Intraurban Digital Divide Using Online Restaurant Reviews: A Case Study in Franklin County, Ohio. The Professional Geographer, 66(3), 443-455. http://doi.org/10.1080/00330124.2013.866431 
boyd, D., \& Crawford, K. (2012). CRITICAL QUESTIONS FOR BIG DATA. Information, Communication \& Society, 15(5), 662-679.

http://doi.org/10.1080/1369118X.2012.678878

Cattell, R. (2011). Scalable SQL and NoSQL data stores. ACM SIGMOD Record, 39(4), 12-27. http://doi.org/10.1145/1978915.1978919

Chew, F. (2016). Using Tweets to Study Empire. Paper presented at the Broadcast

Education Association Conference.

Chodorow, K. (2013). MongoDB: the definitive guide. O'Reilly Media, Inc.

Christian, J. (2014, November). \# epidemiology: Ecological analysis of fast food tweets in relation to Behavioral Risk Factor Surveillance System data. In 142nd APHA Annual Meeting and Exposition (November 15-November 19, 2014). APHA.

Congress, L. O. (2013). Update on the Twitter Archive At the Library of Congress (Vol. 36, pp. 189-198). Library of Congress.

Crampton, J. W., Graham, M., Poorthuis, A., Shelton, T., Stephens, M., Wilson, M. W., \& Zook, M. A. (2013). Beyond the geotag: situating "big data" and leveraging the potential of the geoweb. Cartography and Geographic Information Science, 40(2), 130-139. http://doi.org/10.1080/15230406.2013.777137

Croitoru, A., Crooks, A., Radzikowski, J., \& Stefanidis, A. (2013). Geosocial gauge: a system prototype for knowledge discovery from social media. International Journal of Geographical Information Science, 27(12), 2483-2508. http://doi.org/10.1080/13658816.2013.825724

Devogele, T., Parent, C., \& Spaccapietra, S. (1998). On spatial database integration. International Journal of Geographical Information Science, 12(4), 335-352. http://doi.org/10.1080/136588198241824

Dooms, S., \& De Pessemier, T. (2013). Movietweetings: a movie rating dataset collected from twitter. Presented at the ... on Crowdsourcing and ....

Dugundji, E. R., Poorthuis, A., \& van Meeteren, M. (2011). Modeling User Behavior in Adoption and Diffusion of Twitter Clients. 2011 IEEE Third Int"l Conference on Privacy, Security, Risk and Trust (PASSAT) / 2011 IEEE Third Int"l Conference on Social Computing (SocialCom) (pp. 1372-1379). IEEE. http://doi.org/10.1109/PASSAT/SocialCom.2011.95

Easy, proactive monitoring of processes, programs, files, directories, filesystems and hosts | Monit. (n.d.). Easy, proactive monitoring of processes, programs, files, directories, filesystems and hosts | Monit. Retrieved August 13, 2015, from https://mmonit.com/monit/

Export Twitter Followers and Friends using a Google Spreadsheet. (2011). Export Twitter Followers and Friends using a Google Spreadsheet.

Fisher, E. (2014). Making the most detailed tweet map ever. 
Fujita, H. (2013). Geo-tagged Twitter collection and visualization system. Cartography and Geographic Information Science, 40(3), 183-191. http://doi.org/10.1080/15230406.2013.800272

Gates, A. F., Natkovich, O., Chopra, S., Kamath, P., Narayanamurthy, S. M., Olston, C., et al. (2009). Building a high-level dataflow system on top of Map-Reduce: the Pig experience. Proceedings of the VLDB Endowment, 2(2), 1414-1425. http://doi.org/10.14778/1687553.1687568

Geohashes. (n.d.). Geohashes. Retrieved August 16, 2015, from https://www.elastic.co/guide/en/elasticsearch/guide/current/geohashes.html

Goodspeed, Robert. 2013. "The Limited Usefulness of Social Media and Digital Trace Data for Urban Social Research." Seventh International AAAI Conference on Weblogs and Social Media. Inc.

Gormley, C., \& Tong, Z. (2015). Elasticsearch: The Definitive Guide. O'Reilly Media,

Han, J., Koperski, K., Stefanovic, N., Han, J., Koperski, K., \& Stefanovic, N. (1997). GeoMiner: a system prototype for spatial data mining. ACM SIGMOD Record (Vol. 26, pp. 553-556). ACM. http://doi.org/10.1145/253262.253404

Hill, Z., \& Humphrey, M. (2009). A quantitative analysis of high performance computing with Amazon's EC2 infrastructure: The death of the local cluster? (pp. 26-33). Presented at the 2009 10th IEEE/ACM International Conference on Grid Computing (GRID), IEEE. http://doi.org/10.1109/GRID.2009.5353067

Huang, Q., \& Xu, C. (2014). A data-driven framework for archiving and exploring social media data. Annals of GIS, 20(4), 265-277. http://doi.org/10.1080/19475683.2014.942697

Jung, J.-K. (2015). Code clouds: Qualitative geovisualization of geotweets. The Canadian Geographer / Le Géographe Canadien, 59(1), 52-68. http://doi.org/10.1111/cag.12133

Juve, G., Deelman, E., Berriman, G. B., Berman, B. P., \& Maechling, P. (2012). An Evaluation of the Cost and Performance of Scientific Workflows on Amazon EC2. Journal of Grid Computing, 10(1), 5-21. http://doi.org/10.1007/s10723-012-9207-6

Juve, G., Deelman, E., Vahi, K., Mehta, G., Berriman, B., Berman, B. P., \& Maechling, P. (2009). Scientific workflow applications on Amazon EC2 (pp. 59-66). Presented at the 2009 5th IEEE International Conference On E-Science Workshops, IEEE. http://doi.org/10.1109/ESCIW.2009.5408002

Kay, S., Zhao, B., \& Sui, D. (2014). Can Social Media Clear the Air? A Case Study of the Air Pollution Problem in Chinese Cities. The Professional Geographer, 1-13. http://doi.org/10.1080/00330124.2014.970838

Kitchin, R. (2014). The data revolution: Big data, open data, data infrastructures and their consequences. Sage. 
Klein, K. R. (2014). Tracking a wildfire in areas of high relief using volunteered geographic information: A viewshed application. ProQuest Dissertations and Theses. The University of Utah, Ann Arbor.

Korson, C. (2014). Political Agency and Citizen Journalism: Twitter as a Tool of Evaluation. The Professional Geographer, 1-10.

http://doi.org/10.1080/00330124.2014.970839

Kounadi, O., Lampoltshammer, T. J., Groff, E., Sitko, I., \& Leitner, M. (2015). Exploring Twitter to Analyze the Public's Reaction Patterns to Recently Reported Homicides in London. Plos One, 10(3), e0121848-17. http://doi.org/10.1371/journal.pone.0121848

Kumar, S., Barbier, G., Abbasi, M. A., \& Liu, H. (2011). TweetTracker: An Analysis Tool for Humanitarian and Disaster Relief, 1-2.

Kumar, S., Morstatter, F., \& Liu, H. (2014). Twitter Data Analytics. New York, NY: Springer New York. http://doi.org/10.1007/978-1-4614-9372-3

Laney, Doug. 2001. "3D data management: Controlling data volume, velocity and variety." META Group Research Note 6:70.

Leetaru, K., Wang, S., Cao, G., Padmanabhan, A., \& Shook, E. (2013). Mapping the global Twitter heartbeat: The geography of Twitter. First Monday, 18(5), 290-307. http://doi.org/10.1287/orsc.1050.0122

Levenshtein, V. (1966). Binary Codes Capable of Correcting Deletions, Insertions and Reversals. soDiet. Soviet Physics-Doklady, 10(8). http://doi.org/10.1007/BF00053379

Levy, S. (1984). Hackers: Heroes of the Computer Revolution. New York: Doubleday.

Li, L., Goodchild, M. F., \& Xu, B. (2013). Spatial, temporal, and socioeconomic patterns in the use of Twitter and Flickr. Cartography and Geographic Information Science, 40(2), 61-77. http://doi.org/10.1080/15230406.2013.777139

Lin, J., \& Cromley, R. G. (2015). Evaluating geo-located Twitter data as a control layer for areal interpolation of population. Applied Geography, 58(C), 41-47.

http://doi.org/10.1016/j.apgeog.2015.01.006

MacEachren, A. M., Robinson, A. C., \& Jaiswal, A. (2011). Geo-twitter analytics: Applications in crisis management. 25th International ....

Morstatter, F., Pfeffer, J., Liu, H., \& Carley, K. M. (2013, June 21). Is the Sample Good Enough? Comparing Data from Twitter"s Streaming API with Twitter”s Firehose.

Natural Earth. (n.d.). Natural Earth. Retrieved August 15, 2015, from http://www.naturalearthdata.com/

Olken, F., \& Rotem, D. (1990). Random sampling from database files: A survey. In Statistical and Scientific Database Management (Vol. 420, pp. 92-111). Berlin, Heidelberg: Springer Berlin Heidelberg. http://doi.org/10.1007/3-540-52342-1_23

Oreskovic, A. (2015). Here's another area where Twitter appears to have stalled: tweets per day. Retrieved August 15, 2015, from http://www.businessinsider.com/twittertweets-per-day-appears-to-have-stalled-2015-6 
Ostermann, S., Iosup, A., Yigitbasi, N., Prodan, R., Fahringer, T., \& Epema, D. (2010). A Performance Analysis of EC2 Cloud Computing Services for Scientific Computing. In Cloud Computing (Vol. 34, pp. 115-131). Berlin, Heidelberg: Springer Berlin Heidelberg. http://doi.org/10.1007/978-3-642-12636-9_9

Pokorny, J. (2013). NoSQL databases: a step to database scalability in web environment. International Journal of Web Information Systems, 9(1), 69-82. http://doi.org/10.1108/17440081311316398

Poorthuis, A., \& Zook, M. (2014). Artists and Bankers and Hipsters, Oh My! Mapping Tweets in the New York Metropolitan Region. Cityscape: a Journal of Policy Development and ....

Poorthuis, A., \& Zook, M. (2015). Small Stories in Big Data: Gaining Insights From Large Spatial Point Pattern Datasets. Cityscape: a Journal of Policy Development and ....

Poorthuis, A., Zook, M. A., Shelton, T., Graham, M., \& Stephens, M. (2016). Using Geotagged Digital Social Data in Geographic Research. In N. Clifford, S. French, M. Cope, \& S. Gillespie (Eds.), Key Methods in Geography (3rd ed.).

Ramsey, P. (2005). PostGIS manual. Refractions Research Inc.

Removal of Export and Download / API Capabilities. (2011, February 22). Removal of Export and Download / API Capabilities. Retrieved August 13, 2015, from https://twapperkeeper.wordpress.com/2011/02/22/removal-of-export-and-downloadapi-capabilities/

Schaefer, B. (2014). Social Media to Locate Urban Displacement: Assessing the Risk of Displacement Using Volunteered Geographic Information in the City of Los Angeles. Los Angeles.

Schweitzer, Lisa. 2014. "Planning and Social Media: A Case Study of Public Transit and Stigma on Twitter. "Journal of the American Planning Association 80 (3):218-238. doi: 10.1080/01944363.2014.980439.

Shelton, T. (2015, September 6). Geographies of Data: Toward a Relational SocioSpatial Analysis of Geotagged Social Media Data. Worcester.

Shelton, T., Poorthuis, A., \& Zook, M. A. (2015). Social media and the city: Rethinking urban socio-spatial inequality using user-generated geographic information. Landscape and Urban Planning, 1-14. http://doi.org/10.1016/j.landurbplan.2015.02.020

Shelton, T., Poorthuis, A., Graham, M., \& Zook, M. A. (2014). Mapping the data shadows of Hurricane Sandy: Uncovering the sociospatial dimensions of "big data." Geoforum, 52, 167-179. http://doi.org/10.1016/j.geoforum.2014.01.006

Shelton, T., Zook, M. A., \& Graham, M. (2012). The Technology of Religion: Mapping Religious Cyberscapes. The Professional Geographer, 64(4), 602-617. http://doi.org/10.1080/00330124.2011.614571 
Smith, L., Liang, Q., James, P., \& Lin, W. (2015). Assessing the utility of social media as a data source for flood risk management using a real-time modelling framework. Journal of Flood Risk Management, n/a-n/a. http://doi.org/10.1111/jfr3.12154

Smith, M. A., Shneiderman, B., Milic-Frayling, N., Mendes Rodrigues, E., Barash, V., Dunne, C., et al. (2009). Analyzing (social media) networks with NodeXL (pp. 255-264). Presented at the the fourth international conference, New York, New York, USA: ACM. http://doi.org/10.1145/1556460.1556497

Stefanidis, A., Cotnoir, A., Croitoru, A., Crooks, A., Rice, M., \& Radzikowski, J. (2013). Demarcating new boundaries: mapping virtual polycentric communities through social media content. Cartography and Geographic Information Science, 40(2), 116-129. http://doi.org/10.1080/15230406.2013.776211

Stephens, M. (2012, August 8). FROM GEO-SOCIAL TO GEO-LOCAL: THE FLOWS AND BIASES OF VOLUNTEERED GEOGRAPHIC INFORMATION. Tucson.

The story of getting Twitter data and its "missing middle" | ScraperWiki. (2014). The story of getting Twitter data and its "missing middle" | ScraperWiki. Retrieved August 13, 2015, from https://blog.scraperwiki.com/2014/08/the-story-of-getting-twitter-data-andits-missing-middle/

tweetstream. (2015, September 1). tweetstream/tweetstream. Retrieved August 16, 2015, from https://github.com/tweetstream/tweetstream

Twitter. (2013, August 16). New Tweets per second record, and how! | Twitter Blogs. Retrieved September 21, 2015, from https://blog.twitter.com/2013/new-tweetsper-second-record-and-how

Twitter. (2015, May 18). Developer Agreement \& Policy. Retrieved September 21, 2015, from https://dev.twitter.com/overview/terms/agreement-and-policy

van Meeteren, M., Poorthuis, A., \& Dugundji, E. (2009). Mapping communities in large virtual social networks: Using Twitter data to find the Indie Mac community (pp. 1-8). Presented at the Engaging Data, IEEE. http://doi.org/10.1109/BASNA.2010.5730297

Vinoski, S. (2006). Advanced Message Queuing Protocol. IEEE Internet Computing, 10(6), 87-89. http://doi.org/10.1109/MIC.2006.116

Wang, S. (2010). A CyberGIS Framework for the Synthesis of Cyberinfrastructure, GIS, and Spatial Analysis. Annals of the Association of American Geographers, 100(3), 535557. http://doi.org/10.1080/00045601003791243

Weller, K. (2014). Twitter Und Wahlen. In R. Reichert (Ed.), Big Data. Bielefeld.

White, J., \& Roth, R. E. (2010). TwitterHitter: Geovisual analytics for harvesting insight from volunteered geographic information. Presented at the Proceedings of GIScience.

White, T. (2012). Hadoop: The definitive guide. O'Reilly Media, Inc.

Widener, M. J., \& Li, W. (2014). Using geolocated Twitter data to monitor the prevalence of healthy and unhealthy food references across the US. Applied Geography, 54, 189-197. http://doi.org/10.1016/j.apgeog.2014.07.017 
Xu, C., Wong, D. W., \& Yang, C. (2013). Evaluating the "geographical awareness" of individuals: an exploratory analysis of twitter data. Cartography and Geographic Information Science, 40(2), 103-115. http://doi.org/10.1080/15230406.2013.776212

Yang, W., \& Mu, L. (2015). GIS analysis of depression among Twitter users. Applied Geography, 60, 217-223. http://doi.org/10.1016/j.apgeog.2014.10.016

Zimmer, M., \& Proferes, N. J. (2014). A topology of Twitter research: disciplines, methods, and ethics. Aslib Journal of Information Management, 66(3), 250-261. http://doi.org/10.1108/AJIM-09-2013-0083

Zook, M. (2017). Crowd-sourcing the Smart City: Using Big Geosocial Media Metrics in Urban Governance. Big Data and Society. Forthcoming.

Zook, M. A., \& Poorthuis, A. (2014). Offline Brews and Online Views: Exploring the Geography of Beer Tweets. In The Geography of Beer (pp. 201-209). Dordrecht: Springer Netherlands. http://doi.org/10.1007/978-94-007-7787-3_17

Zook, M., Poorthuis, A, Donohue, R. (2016). Mapping Spaces: Cartographic Representations of Online Data. Chapter for the Handbook of Online Research Methods second edition 